\title{
Le Consortium forestier et maritime des chemins de fer français
}

\section{Édouard Perret}

\section{CpenEdition}

\section{Journals}

Édition électronique

URL : https://journals.openedition.org/rhcf/1864

DOI : 10.4000/rhcf.1864

\section{Éditeur}

Rails \& histoire

\section{Édition imprimée}

Date de publication : 1 juin 2003

Pagination : 61-76

ISBN : 0996-9403

ISSN : 0996-9403

\section{Référence électronique}

Édouard Perret, "Le Consortium forestier et maritime des chemins de fer français », Revue d'histoire des chemins de fer [En ligne], 27 | 2003, mis en ligne le 13 janvier 2015, consulté le 22 avril 2022. URL http://journals.openedition.org/rhcf/1864 ; DOI : https://doi.org/10.4000/rhcf.1864 


\section{Édouard PERRET}

\section{Le Consortium forestier et maritime des chemins de fer français}

\section{Les origines}

Le premier conflit mondial ayant eu pour effet une exploitation intensive des forêts françaises pour les besoins des armées (baraquements, tranchées étayées) à laquelle s'ajoutait la destruction des forêts de l'Est, les autorités françaises se préoccupèrent, dès le début de 1917, se rechercher des sources nouvelles d'approvisionnement en bois pour satisfaire les besoins des PT'T, des réseaux électriques, des compagnies minières et des compagnies de chemins de fer (principalement en traverses et planches de fond de wagon $)^{1}$.

C'est ainsi qu'une mission ferroviaire, placée sous les ordres du colonel du Génie Salesse, fut chargée en 1917 de procéder à une étude approfondie des possibilités offertes par les zones forestières comprises entre Dakar et Pointe-Noire. Le 22 février 1918, cette mission préconisait le choix du Gabon qui offrait une très grande variété d'essences de bois durs susceptibles d'être transformées en traverses. Adoptant les conclusions de ce rapport, les compagnies ferroviaires fondèrent en 1920 le «Consortium des grands réseaux français » (CGRF) ayant pour objet l'exploitation d'une concession forestière (avec industries connexes), accordée pour une durée de 30 ans par décret gouvernemental et approuvée par les autorités françaises de tutelle du Gabon.

Cette concession, de 120000 hectares, qui comportait deux zones distinctes, dénommées Igombiné et Maga, se situait au sud de l'estuaire du Gabon à une centaine de kilomètres de Libreville (voir fig. 1). Le point d'origine de ces deux zones était basé sur une rivière navigable qui permettait l'évacuation des traverses produites vers leur lieu d'embarquement pour la France, sur des navires affrétés par les compagnies de chemins de fer, ainsi que le ravitaillement en vivres des chantiers. La gestion administrative et financière du consortium était

1- Cette note a été présentée le 13 mai 1993 davant la Commission " Histoire des Installations fixes ». M. Alias, son président, a étudié les rapports d'activité du Consortium : son analyse figure en annexe. M. Jean-Claude Gross, ancien directeur du CFM de 1967 à 1972, a ajouté au texte des précisions qui figurent à partir de la note 2, sauf mention contraire. 


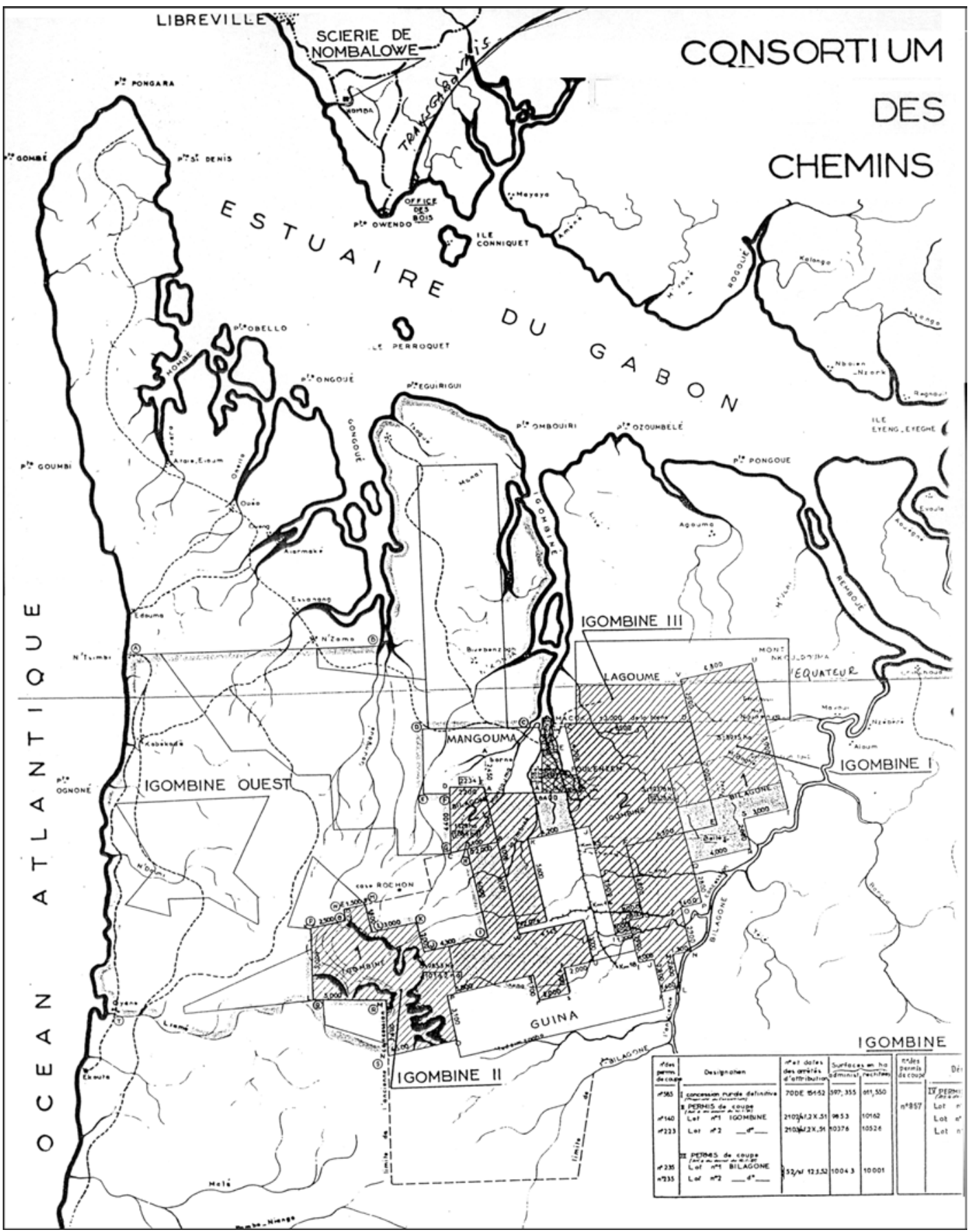

Figure 1. SNCF, direction de l'Équipement, Carte des concessions forestières du consortium forestier et maritime des chemins de fer français, mise à jour le 10 octobre 1967. 


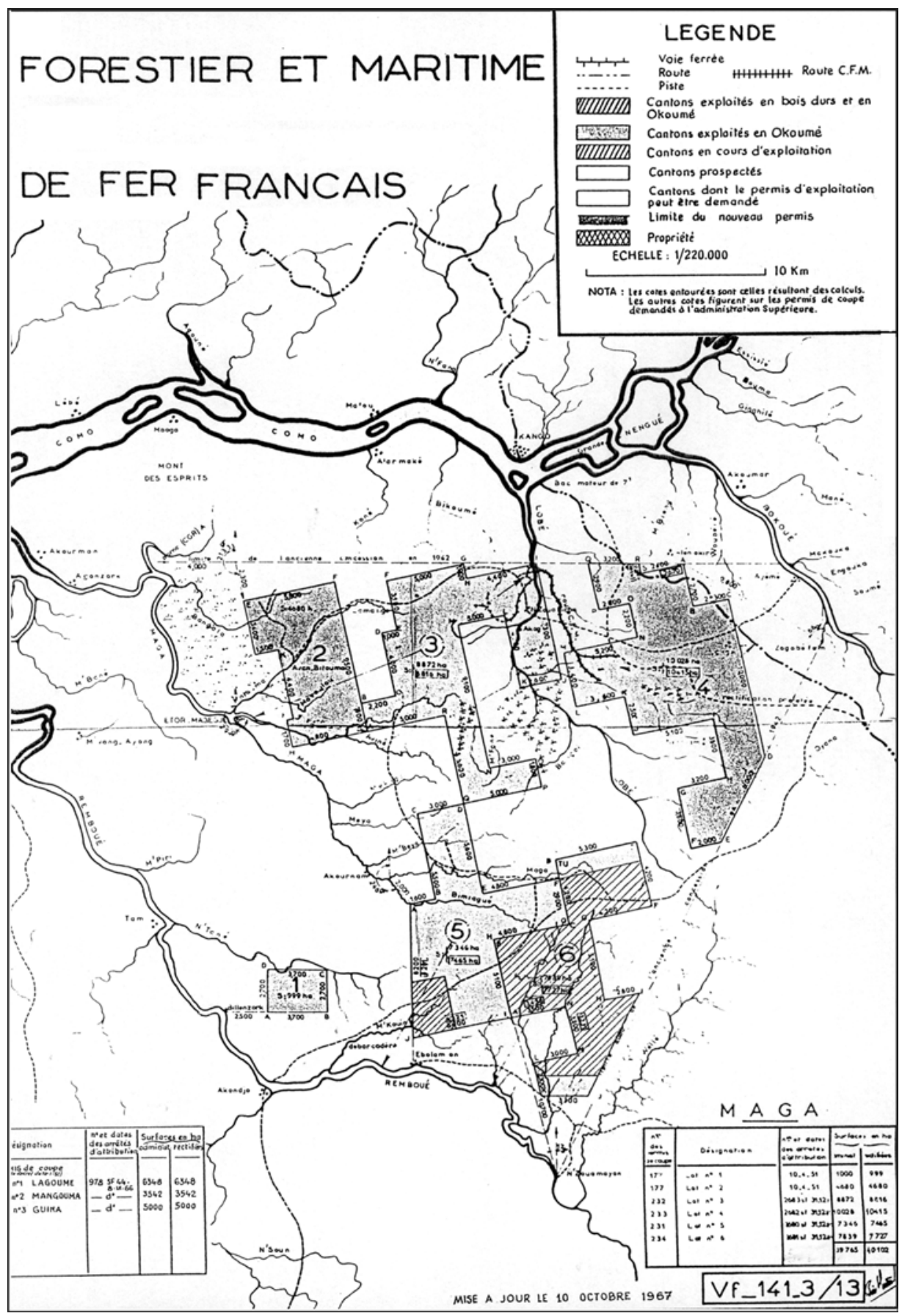


assurée par un comité de direction, composé d'un haut représentant désigné par chaque compagnie. Lors de la création de la SNCF, en 1937, le consortium devint tout naturellement un service annexe de la toute jeune société nationale et prit le nom de « Consortium forestier et maritime des chemins de fer français ", plus communément désigné par le sigle CFM. Doté de l'autonomie financière mais sans personnalité juridique distincte, le CFM était contrôlé par un comité de gestion placé sous la présidence d'un membre désigné du conseil d'administration de la SNCF, assisté du secrétaire général adjoint de la SNCF. Était membre de droit de ce comité le directeur de chacun des grands services d'alors (Équipement, Matériel, Finances, Approvisionnements). Un rôle prépondérant était confié à la direction de l'Équipement, qui assurait la préparation et le suivi des budgets d'investissements et d'exploitation, le recrutement et la gestion du personnel d'encadrement français en service au Gabon, les achats et expéditions du matériel et des approvisionnements nécessaires en collaboration avec le service Achats, les réceptions et répartitions des bois sous rails livrés du Gabon. La direction Finances assurait la comptabilité et la présentation de tous les documents comptables et de trésorerie. Le comité de gestion se réunissait plusieurs fois par an pour approuver les budgets et les résultats comptables, contrôler la marche du CFM, etc., et présentait un rapport d'ensemble bisannuel au conseil d'administration de la SNCF.

\section{Aperçu sur le Gabon}

Le Gabon, qui tire son nom d'un mot portugais signifiant « caban de marin $»^{2}$, a une superficie de $267000 \mathrm{~km}^{2}$ et est peu peuplé d'environ 1200000 habitants $^{3}$ (dont $400000^{4}$ rien que pour la capitale Libreville), comprend une cinquantaines d'ethnies. Il faisait partie de l'ex AEF fondée le 15 janvier 1910. En 1960 le principe de l'indépendance des États africains francophones est décidé et, le 17 août 1960, le Gabon devient un État indépendant sous le nom de République gabonaise et est admis à siéger à L'ONU le 20 septembre 1960. La langue officielle est le français.

Situé de part et d'autre de l'Équateur, le pays a un climat caractérisé par l'alternance de courtes saisons sèches (quatre mois par an) et par des saisons des pluies très importantes entrainant une pluviosité comprise entre 3,50 m et 4,50 m par an. Le Gabon est de ce fait irrigué

2- Selon d'autres étymologies, «petite crevette ».

3- Chiffre 1997. Densité 4,5 ha/ $\mathrm{km}^{2}$.

4- Estimation pour 1999. 
par un réseau hydrographique extrêmement dense qui apporte une ponctuation dans la végétation d'une extraordinaire densité et souvent rendue impénétrable à la fois par les lianes et par les marigots. La température varie de $18^{\circ}$ à $30^{\circ}$, mais le taux d'humidité atteint de 75 à $95 \%$ suivant les saisons, ce qui rend le climat difficile à supporter et très agressif vis-à-vis des engins mécaniques utilisés par les exploitants forestiers dont les activités sont également très perturbées par l'abondance des pluies équatoriales (inondations, routes défoncées...).

En dehors de cet immense potentiel forestier (22 millions d'hectares), le sous-sol renferme des ressources importantes (manganèse, fer, uranium notamment) et des gisements de pétrole, sur terre et offshore, ont été décelés et mis en exploitation depuis 1956. Des industries annexes (barrages et usines hydroélectriques, raffineries, usines de traitement chimique) on été créées. Enfin, pour compenser la faiblesse du réseau routier due au climat et à la nature du sol, très argileux, le gouvernement gabonais a décidé en 1974 la création d'une infrastructure ferroviaire : le Transgabonais. Celui-ci, d'une longueur de $650 \mathrm{~km}$ à écartement de $1,437 \mathrm{~m}$ avec rails de $60 \mathrm{~kg} / \mathrm{m}$ sur traverses en bois (azobé), a permis le désenclavement des régions forestières et minières de l'intérieur, contribué efficacement au développement économique et touristique du pays et a métamorphosé les rapports humains et la notion même de distance. Il constitue à cet égard la véritable épine dorsale du Gabon. La SNCF a participé activement, par l'intermédiaire de SOFRERAIL, de ses ingénieurs et techniciens, à l'étude du projet tant technique que financier, à la définition du tracé définitif de la ligne avec ses très nombreux ouvrages d'art et à sa réalisation.

\section{Le consortium}

Dès 1920, le consortium entreprend la mise en place d'une exploitation forestière dans la zone Igombiné. La zone Maga ne sera en fait exploitée, d'ailleurs essentiellement en bois tendres (okoumé, ozigo), qu'à partir de 1948 pour des raisons de grand éloignement, de difficultés techniques liées à la présence d'une véritable mangrove et à la rareté d'essences de bois durs.

\section{Organisation d'origine : période de 1920 à 1946}

\section{Libreville (quartier de Glass)}

Sur un terrain de quelques hectares concédé en même temps que les zones d'exploitation, une antenne légère a été installée à Libreville avec pour rôle d'assurer toutes les opérations nécessaires à une exploitation industrielle (réception des matériels, des approvisionnements, des 
vivres et du courrier, leur réexpédition vers l'exploitation, opérations de douane tant import qu'export, relations avec les autorités et les banques locales). Cette antenne était dirigée par un transitaire secondé par une trentaine d'employés gabonais.

\section{Macok (rocher, en langue gabonaise)}

Situé à la naissance de la rivière Igombiné, navigable en toutes saisons, le permis forestier a pour point d'origine le lieu-dit Macok, à une centaine de $\mathrm{km}$ au sud-est de Libreville, très près de la ligne de l'équateur. La nature du sol, constitué de larges rochers plats, permet l'édification d'un petit port ( $w h a r f$, quais d'appontement équipés de grues légères, grande grue pivotante avec flèche de $20 \mathrm{~m}$ fonctionnant grâce à une imposante chaufferie à vapeur, atelier d'entretien). La flottille, comprenant deux gros remorqueurs à vapeur, trois pinasses légères et plusieurs chalands de 100 tonnes, mouille à quelque cinquante mètres du port.

Macok devient dès 1920 le siège de la direction de l'exploitation. Un village est édifié, composé des bureaux, des logements du personnel français et gabonais, soit en tout une de centaine de personnes avec leur famille. En 1953, l'installation d'une légère station de radio a permis d'améliorer sensiblement les relations avec l'antenne de Libreville. En effet, la traversée Macok-Libreville représente des trajets de 80 à $100 \mathrm{~km}$ qui sont effectués au minimum en trois heures en utilisant sur la partie maritime du trajet des pinasses rapides et au maximum en deux jours avec des remorqueurs tractant des rideaux de bois ou des chalands chargés, en tenant compte des marées très sensibles en zone équatoriale.

\section{Foulenzem (N'foulenzem, croisée des chemins, en langue gabonaise)}

A $6 \mathrm{~km}$ au sud de Macok, le lieu-dit Foulenzem, constitué d'un large plateau de plusieurs dizaines d'hectares, se prêtait bien à l'implantation du centre industriel. Dès 1922, après déforestage, une scierie principale y est installée, équipée de scies alternatives verticales à lames multiples, d'une dédoubleuse à ruban, d'une déligneuse horizontale de grande longueur, de plusieurs tronçonneuses circulaires à balancier. Une scierie seconde s'y ajouta pour le débitage de planches de fond de wagon et des débits spéciaux. Elle était constituée de deux scies à ruban Panhard de 1,10 $\mathrm{m}$ et 1,50 $\mathrm{m}$ de diamètre avec déligneuse à lames multiples. 
L'approvisionnement en bois des scies fut assuré par un portique roulant amenant les grumes, stockées sur un parc de deux hectares et de $10000 \mathrm{~m}^{3}$ de capacité totale. Des aires importantes furent réservées au stockage des produits fabriqués avant leur évacuation sur Macok puis leur expédition vers les navires-chargeurs stationnant près de l'île des Perroquets située à l'embouchure de l'Igombiné.

Le centre industriel fut complété par une série d'ateliers annexes : affûtage, mécanique générale et à vapeur, mécanique moteurs, menuiserie bien équipée, magasin général d'outillage et de pièces de rechange, station de pompage d'eau dans la rivière Foulenzem toute proche et non salée. L'énergie nécessaire était fournie par une centrale thermique alimentée au bois. Elle fut remplacée en 1947 par trois chaudières de $125 \mathrm{~m}^{2}$ alimentées également au bois et entrainant une génératrice par l'intermédiaire d'une machine à vapeur de $250 \mathrm{CV}$. Deux moteurs diesel et trois alternateurs de 300 CV s'ajoutaient à ce nouvel équipement.

Un dispensaire avec salle chirurgicale, une école pour les enfants gabonais et une gendarmerie complétaient cet ensemble qui devint dès cette époque une bourgade de 4000 habitants entièrement centrée sur les activités du consortium.

Parallèlement, pour assurer l'approvisionnement du centre et l'évacuation des grumes et des produits de sciage, un chemin de fer (qui fut longtemps le seul au Gabon) à voie de 0,50 $\mathrm{m}$ fut implanté et desservi par plusieurs locomotives à vapeur Decauville, une centaine de wagons plats avec ranchers, quelques wagons tombereaux et draisines légères à bras. Cette ligne à voie unique desservit, en premier lieu, le trajet Macok-Foulenzem avec de nombreux épis et évitements tant à Macok que dans le centre industriel.

Enfin, à l'est de ce centre, fut construit un village de logements individuels pour le personnel et, à l'ouest, une plantation, couvrant plusieurs hectares, permettait de fournir en produits frais une partie de l'alimentation du personnel et des familles (bananes, citrons, papayes, cocotiers, ananas, avocatiers, palmiers à huile...).

\section{Les chantiers forestiers}

La forêt gabonaise, très dense, est peuplée d'environ 800 espèces dont 200 sont bien connues et utilisables grâce aux études menées par les ingénieurs spécialistes du Centre technique forestier tropical, organisme d'État dépendant du ministère de l'Agriculture. En général très droits, lisses et nus, d'une circonférence pouvant atteindre plusieurs mètres, ces arbres, implantés dans le sol par d'énormes racines horizontales raccordées aux troncs par des contreforts atteignant souvent 
$2 \mathrm{~m}$ de hauteur, se terminent par un houppier couronnant l'arbre à 20, 30 et même $40 \mathrm{~m}$ au dessus du sol. C'est la forêt vierge primaire à l'espace relativement dégagé au niveau du sol mais où règne en permanence une semi-obscurité. Après abattage de ces géants, la lumière peut enfin pénétrer au niveau du sol et permettre la régénérescence des essences jusqu'alors étouffées. C'est la forêt secondaire, moins haute mais où les sous-bois sont le plus souvent infranchissables sans un important travail de défrichage, en raison du développement d'une végétation luxuriante et parasitaire (lianes, fougères arborescentes, herbes de toutes espèces, fleurs).

Dès 1923, le consortium entreprit de prospecter la concession Igombiné au sud de Foulenzem. Les équipes sont chargées de diviser et de jalonner le terrain en carrés de $250 \mathrm{~m}$ de côté, d'en dresser la carte en y précisant le relief et la position des cours d'eau et de recenser les essences exploitables, distinguées en bois tendres (okoumé, ozigo, afo...), bois nobles d'exportation (acajou, noyer...) et bois durs à traverses (une vingtaine d'espèces admises par les chemins de fer, principalement bilinga, azobé, iroko, moabi, douka, padouk). Le chef d'exploitation rassemble ensuite ces données et dresse une carte générale lui permettant de déterminer le tracé des voies d'évacuation des bois (principalement par voie ferrée) et le cubage approximatif des arbres de chaque catégorie.

La concession Igombiné fut divisée en plusieurs lots (voir fig. 1). Trois chantiers distincts eurent pour mission l'exploitation totale des ressources utilisables, qui était une clause impérative du décret de concession. Chacun d'eux comprenait environ 100 hommes sous la conduite d'un chef de chantier, assisté d'un mécanicien. Le personnel était divisé en 5 groupes : abattage, tronçonnage des fûts en rondins de longueurs appropriées à leur usage, tracé et pose de la voie ferrée sur fascines, défrichage du terrain entre le parc de tronçonnage et la voie ferrée, chargement sur les wagons plats et évacuation sur Foulenzem (bois à traverses) ou vers l'embarcadère de Macok (bois tendres flottables) où ils étaient mis à l'eau et rassemblés en radeaux.

Il convient de souligner que, jusqu'en 1946, les travaux s'effectuaient dans des conditions particulièrement pénibles compte tenu des efforts demandés sous un climat hostile aggravé par la présence de myriades d'insectes, vecteurs de germes de maladies graves (paludisme, filariose, trypanosomiase). L'abattage s'effectuait à la hache, le tronçonnage du houppier du fût à la scie passe-partout, le défrichage du terrain à la machette, le poussage des rondins vers la voie ferrée d'évacuation au moyen de bambous, l'arrimage à la liane sur les wagons, le tout réalisé huit mois par an sous des pluies torrentielles. Dans ces 
conditions, il est certain que le consortium n'exploita que les parcelles relativement faciles d'accès et riches en essences recherchées.

\section{Conclusion}

Durant cette période de 1920 à 1946, l'évolution de la production a été très irrégulière compte tenu des difficultés rencontrées : climat, main-d'œuvre locale instable et insuffisante, terrain d'accès malaisé, pénurie de pièces de rechange, inexistence de matériels mécaniques efficaces, éloignement de la France pour les approvisionnements (délai d’au moins 4 mois pour toute commande de matériel ou de pièces).

Sil'on relève une commercialisation annuelle de près de $25000 \mathrm{~m}^{3}$ de grumes d'okoumé, bois de densité 0,7 (bois de déroulage par excellence destinés à la fabrication du contre-plaqué) ${ }^{5}$, on note une production annuelle de traverses variant de 7000 à $12000 \mathrm{~m}^{3}\left(10\right.$ traverses au m $\left.\mathrm{m}^{3}\right)$. L'effectif s'élève à une trentaine d'agents européens (principalement des Français et des Piémontais) et environ 600 ouvriers africains. Il faut rappeler qu'en ce qui concerne les Européens, la durée de leur séjour ne pouvait excéder 30 mois suivis de 6 mois de congé en Europe pour des raisons de santé (malnutrition, fatigue excessive, inexistence de produits pharmaceutiques appropriés aux risques encourus).

Une parenthèse se produisit entre les années 1940 et 1944 du fait du conflit mondial. Étant coupé de la France, le consortium dut subvenir à ses besoins sur le monde africain (Afrique du Sud principalement). Nombre d'Européens furent mobilisés et mutés dans des unités de maintenance (Afrique du Sud, Congo, Cameroun). Une base importante d'hydravions et de vedettes rapides de la RAF s'étant constituée dans la baie d'Owendo, à $20 \mathrm{~km}$ à l'est de Libreville sur la même rive de l'estuaire, la production de la scierie de Foulenzem, seule scierie existant au Gabon, fut totalement absorbée pour la construction de cette importante base militaire qui avait pour mission la protection du trafic maritime entre le Congo et le Golfe de Guinée et la lutte contre les sous-marins et bâtiments de guerre allemands.

\section{Évolution}

A partir de 1946, le développement des techniques et du matériel modifie profondément les activités forestières au Gabon ; s'y ajoutent la reprise des relations commerciales internationales, la desserte bi-hebdomadaire du Gabon par avion, l'amélioration sensible des conditions d'existence (groupes électrogènes, réfrigérateurs, stations privées de radio, apport régulier de vivres frais) et l'apparition d'une médecine prophylactique utilisant les dernières découvertes médicales : nivaquine, antibiotiques, etc.

5- ...et en faible partie d'ozigo. 


\section{Période 1946-1960}

Le consortium, ayant demandé et obtenu le renouvellement de ses concessions forestières dont la superficie fut toutefois volontairement limitée à 90000 hectares par l'abandon des zones totalement exploitées depuis 1920, procède alors à la modernisation indispensable de l'ensemble de ses secteurs d'activité.

Chaque chantier forestier, auquel s'ajoute celui ouvert en Maga, est doté de 4 tracteurs à chenilles avec treuil et pelle, de plusieurs tronçonneuses thermiques à haut rendement. Le secteur de la voie reçoit quatre locotracteurs Diesel et une centaine de nouveaux wagons plats. La flottille est renforcée par deux gros remorqueurs Diesel, six plates et deux pinasses rapides. Une station émettrice-réceptrice de radio est installée tant à Libreville qu'à Macok. La scierie de Foulenzem s'équipe de nouvelles scies alternatives et à ruban et de moyens modernes de manutention. Le dispensaire se transforme en hôpital de campagne avec salle de chirurgie, pharmacie, radiologie et hébergement, sous la conduite d'un médecin-chirurgien français.

Toutefois, pour assurer le financement de ces importants investissements, l'accent est porté sur le développement de l'exploitation de l'okoumé, essence très rentable ne nécessitant aucune transformation. C'est ainsi que, entre 1947 et 1960, le consortium livre à l'OBAE (Office des bois de l'Afrique équatoriale situé dans la baie d'Owendo, organisme d'État seul habilité légalement à commercialiser les bois de déroulage) entre 30000 et $35000 \mathrm{~m}^{3}$ de grumes d'Okoumé chaque année. Dans le même temps, les envois de bois sous rails à la SNCF se limitent à une moyenne de l'ordre de $3200 \mathrm{~m}^{3}$ par an mais, parallèlement, se développe une exportation importante de sciages de bois tendres, en particulier okoumé impropre au déroulage, très demandés par les pays de l'Europe du Nord (Suède, Grande-Bretagne, Pays-Bas) et par ceux de l'Afrique australe (Mozambique, Afrique du Sud, Angola) et une amorce de ventes sur le marché local de débits de bois divers de toutes dimensions pour satisfaire les besoins de Libreville en pleine expansion.

Durant cette période, la production moyenne annuelle atteint $75000 \mathrm{~m}^{3}$ de grumes pour l'ensemble des chantiers et environ $8000 \mathrm{~m}^{3}$ de sciage pour la scierie de Foulenzem. A noter que cette production nécessite une extension importante des zones exploitées et une pose accélérée de la voie ferrée qui représentera dès 1950 une longueur d'environ $50 \mathrm{~km}$. 
L'effectif du personnel atteint 35 agents français (près de la moitié de ceux-ci viennent de la SNCF, notamment de l'Atelier du Moulinneuf) et de près de 1000 autochtones. Un important effort de formation est mené et de très nombreux Gabonais acquièrent la qualité d'ouvriers qualifiés et même d'agents de maitrise, scieurs, électriciens, menuisiers, charpentiers, contremaitres de chantiers, infirmiers, conducteurs d'engins, comptables, maçons, pilotes de remorqueur, agents en douane.

De même, les conditions de vie s'améliorent notamment pour le personnel autochtone avec la construction de logements individuels mieux adaptés au climat, la fourniture gratuite de couvertures, d'ustensiles de cuisine, de pétrole pour l'éclairage, l'amélioration de la nourriture par l'envoi régulier par les économats de la SNCF de produits de première nécessité (riz, légumes secs, savon), la vaccination des familles, la fourniture de nivaquine, l'extension de l'école avec affectation d'un instituteur gabonais désigné par les autorités de Libreville. Il convient de rappeler qu'il était fait obligation à toute entreprise s'installant au Gabon de subvenir gratuitement aux besoins de son personnel et des familles.

\section{Période 1960 - 1972}

Les années 1960-1972 marquent un tournant dans l'activité du consortium en raison de l'accès du Gabon à l'indépendance. A la demande expresse du gouvernement gabonais, les autorités supérieures de la SNCF acceptent de procéder à l'extension industrielle du consortium. Après des pourparlers longs et délicats, une convention d'établissement décennale est signée en 1962. En échange de certains assouplissements douaniers et fiscaux, la SNCF s'engage à créer et à financer une très importante scierie à Nombalowé ${ }^{6}$ et à rénover les installations de Foulenzem pour porter le potentiel industriel du consortium à une capacité de production de l'ordre de $30000 \mathrm{~m}^{3}$ par an.

La scierie de Foulenzem fut donc entièrement rénovée en 1961 par l'acquisition de trois nouvelles scies alternatives à haut rendement. Une scierie seconde, destinée aux débits spéciaux, fut installée avec une nouvelle grosse scie à ruban à volant de 1,50 $\mathrm{m}$ et une déligneuse à lames multiples. L'ensemble fut complété par une triple chaîne de tirage manuel sur transporteurs à rouleaux. Le parc à grumes fut équipé de loggers qui amenaient directement et rapidement les grumes sur le chariot des scies. Enfin, des tronçonneuses rotatives coupaient à longueur les

6- Située au faubourg de Libreville en direction d'Owendo. 
plateaux sortant des scies. La production de ce centre atteignit, à partir de 1962 et jusqu'en 1972, de 15000 à $25000 \mathrm{~m}^{3}$ de traverses par an (le record fut enregistré en 1968 avec $25092 \mathrm{~m}^{3}$ ).

La nowelle scierie de Nombalowés'installa sur un terrain de 25 hectares attribué en toute propriété par le gouvernement gabonais. Les études de cet ensemble furent menées conjointement avec le CTFT et aboutirent à l'établissement d'une unité de sciage ultra-moderne et réservée en première place au débitage de bois tendres (okoumé principalement).

Amenées par flottaison sur la rivière de Nomba, les billes étaient hissées sur une chaine monte-grume automatique d'une centaine de mètres de longueur qui les posait sur le chariot d'une très grosse scie à ruban à volant de $2,10 \mathrm{~m}$ et lame de $350 \mathrm{~mm}$. Les plateaux débités étaient repris par une scie seconde alternative à lames multiples et tronçonnés à la longueur voulue par une tronçonneuse de grand diamètre. Les sciages ainsi terminés étaient automatiquement triés en fonction de leur longueur grâce à un système mécanique simple mais robuste et efficace. Les sciures et déchets étaient dirigés par un tapis roulant jusqu'à une tour de brûlage. Toutes ces opérations étaient automatiques. L'ensemble était complété par un certain nombre de scies annexes plus légères (déligneuse, abouteuse, dédoubleuse).

La production de cette scierie s'élevait à environ $12000 \mathrm{~m}^{3}$ par an. A l'origine, ces débits étaient orientés vers l'exportation mais le développement économique du pays permit, dès 1965, d'assurer la vente locale de la totalité de la production (à noter qu'une place de plus en plus importante fut faite au débitage d'essences plus nobles telles que bilinga, afo, douka, très appréciées de la clientèle).

Cette unité de production fut officiellement inaugurée en 1963 en présence de $\mathrm{M}$. Léon $\mathrm{Mb}$, président de la République gabonaise, et de M. Pierre Renaud, vice-président du conseil d'administration de la SNCF.

La direction du CFM étudia en 1964 la possibilité de l’utilisation des sous-produits de Nombalowé et c'est ainsi qu'à cette date un important atelier de menuiserie vient s'adjoindre à la scierie. Principalement équipé par le transfert des machines à bois de l'atelier de Foulenzem, cet atelier se spécialisa dans la construction de panneaux de « cases » (ce terme recouvre au Gabon l'ensemble des logements d'habitation quelle que soit leur classe). Cette activité secondaire ne cessa de progresser d'année en année et consommait à elle seule près de $3000 \mathrm{~m}^{3}$ de sciage par an. L'intérêt de la clientèle s'étant porté non plus sur des cases tout en bois 
mais sur des constructions mixtes, le CFM s'allia à un architecte et à un entrepreneur de travaux publics pour offrir, à des prix compétitifs, des bâtiments traditionnels, le bois conservant une place prépondérante pour la décoration et le doublage des murs en béton. De nombreux bâtiments furent ainsi édifiés : lycée, université, résidence présidentielle, écoles, bureaux d'études, immeubles collectifs...

Dans le même temps, il était procédé au remaniement complet des chantiers forestiers pour faire face à l'accroissement des besoins en bois durs et tendres. Or cette production ne pourrait être atteinte qu'en exploitant les zones accidentées délaissées par la gestion précédente faute de matériels adéquats. La décision fut donc prise de remplacer la voie ferrée incapable d'atteindre ces zones par des routes permettant l'utilisation de camions grumiers.

Après dépose des rails, d'ailleurs à limite d'usure, les plates-formes furent élargies pour permettre l'établissement de routes, munies de larges fossés d'écoulement des eaux et largement éclairées afin de faciliter une évaporation rapide. Malheureusement la région étant dépourvue de latérite (matériau idéal qui revêt la majorité des pistes équatoriales), les routes, établies sur terrain argileux ou sablonneux, entrainaient un coût élevé d'entretien par l'emploi continu d'engins de nivellement et la fatigue excessive des convois lesquels, d'ailleurs, ne pouvaient circuler que pendant 170 jours par an. Chaque chantier fut doté de deux excavateurs sur pneus, de six camions grumiers $(6 \times 6)$ de 32 t et de voitures de service tout-terrain qui venaient s'ajouter aux engins déjà en place.

La production escomptée et nécessaire, soit $75000 \mathrm{~m}^{3}$ de grumes par an, fut atteinte. Elle était principalement constituée de bois durs à traverses, les ventes d'okoumé à l'ONBG (Office national des bois du Gabon qui succéda à l'OBAE) chutant de $44000 \mathrm{~m}^{3}$ à $1300 \mathrm{~m}^{3}$ par an. L'okoumé n'entrant pas dans la qualité « déroulage » fut livré à la scierie de Nombalowé.

De son côté, la direction et les services administratifs furent transférés de Macok à Libreville sur le terrain de Glass, propriété du consortium. Ce changement conduisit à la construction sur ce terrain de bureaux et de «cases » pour le logement du personnel européen. De plus, afin d'accélérer les rotations entre Libreville et Foulenzem (vivres, médicaments, courrier, pièces de rechange...), le consortium acquit un avion monomoteur léger basé à l'aéroport de Libreville et qui faisait deux 
fois par semaine la liaison avec Foulenzem où fut établie une piste d'atterrissage rudimentaire par simple nivellement d'une partie du plateau. De plus, un télex fut installé entre Paris et Libreville.

\section{Conclusions générales}

En 1972, la convention d'établissement liant la SNCF au gouvernement gabonais arrivait à expiration. A cette époque, les installations de Foulenzem, conçues pour que leur durée de vie corresponde à celle de la convention, arrivaient à limite d'usure et se trouvaient, en outre, à l'intérieur d'une zone forestière qui, exploitée depuis 1921, était épuisée en essences exploitables.

L'étude de l'évolution du marché des traverses en France et de la compétitivité de la traverse en béton a démontré le peu d'intérêt de poursuivre une telle entreprise d'autant qu'il aurait fallu procéder au renouvellement des gros matériels et solliciter de nouvelles concessions forestières très éloignées des centres de commercialisation. Dans ces conditions, la SNCF a préféré cesser ses activités industrielles au Gabon. Les installations de Libreville et de Nombalowé furent vendues à des entreprises locales qui continuent d'ailleurs à les exploiter dans des conditions analogues à celle du CFM. L'ensemble du matériel restant, tant en forêt qu'à Macok et Foulenzem, fut cédé à des entreprises locales souvent dirigées par des Gabonais. La concession Maga fut reprise par un organisme d'Etat chargé de mettre en place une unité de production de cellulose.

\section{Quelques chiffres}

- le CFM employait en pleine activité environ 1000 agents gabonais et une quarantaine d'Européens ;

- plus de 700 européens ont été employés au CFM de 1921 à 1972 ;

- le chiffre d'affaires du CFM avoisinait, dans la demière période, 1 milliard de francs CFA par an (soit 20 millions de francs français courants).

Les cinquante années de présence de la SNCF au Gabon ont permis de tisser des liens d'amitié avec le peuple gabonais. Elles ont contribué efficacement à l'industrialisation du pays, à la connaissance approfondie des essences de ce pays grâce aux études menées tant par la SNCF que par le CTFT et à la formation professionnelle de plusieurs centaines de Gabonais qui ont retrouvé sans peine un emploi après le départ du CFM. Comme déjà précisé, la SNCF ne fut pas absente du Gabon après 1972. Elle contribua en effet à l'étude et à la réalisation du «Transgabonais », gage de la prospérité future de cette nation. 


\title{
Annexe
}

\section{La SNCF et Le Consortium financier du Gabon}

\author{
Note de synthèse des archives financières du consortium, \\ conservées par la direction de l'Infrastructure de la SNCF
}

Jean Alias, 1998

Le CFM était un service de la SNCF doté de l'autonomie financière et placé sous l'autorité et le contrôle d'un conseil d'administration présidé par un administrateur de la SNCF (M. Le Vert de 1964 à 1974). Les investissements étaient financés par la SNCF qui percevait un intérêt statutaire sur la totalité des découverts, égal au taux directeur de la Banque de France, majoré d'un point. Elle ne se comportait donc pas en actionnaire, mais plutôt en obligataire.

Jusqu'en 1964, l'essentiel de la production était orienté vers l'exploitation et la vente de l'okoumé, richesse principale du Gabon à cette époque, et, accessoirement, vers l'exploitation et la vente de bois durs et l'exportation de traverses à destination de la SNCF. De $25000 \mathrm{~m}^{3} \mathrm{en}$ 1956 la production d'okoumé s'est élevée dans les années suivantes pour culminer à $57100 \mathrm{~m}^{3}$ en 1961 et ensuite diminuer rapidement, du fait de l'épuisement des réserves de la concession (un okoumé demande environ 75 ans pour devenir exploitable) jusqu'à un chiffre voisin de zéro en 1969.

Pour compenser cette perte, aggravée par les lourds investissements de la scierie de Nombalowé en 1964, le CFM a intensifié la production de bois durs vendus en grumes à l'exportation et la production de traverses dans le cadre des besoins de la SNCF, qui les achetait aux prix du marché ; le lancement à partir des années 1970 de constructions en bois préfabriquées a également permis d'améliorer les résultats financiers. Les cubages de sciages annuellement vendus à la SNCF se sont situés dans les dix dernières années entre 15 et $25000 \mathrm{~m}^{3}$ par an, correspondant à plus de 150000 traverses par an ${ }^{7}$ auxquelles s'ajoutaient les bois d'appareil.

Les investissements financés par la SNCF ont été de 320 millions de francs CFA en 1961 et de 910 millions de francs CFA de 1962 à 1972 soit un total de 1522 milions de francs CFA de 1950 à 1972 (ou 30 millions de francs français). Il convient de noter que la parité de

7- Précision apportée par M. Gross. 
1 FFR pour 50 FCFA était surévaluée, et a été récemment ramenée à $1 / 100^{8}$. D'autre part, pendant la période de référence de 1962 à 1974, le franc français et par conséquent le franc $\mathrm{CFA}$ ont perdu près de $50 \%$ de leur valeur. Les chiffres cités sont donc plutôt des ordres de grandeurs.

Depuis sa création (1920) jusqu'en 1962, le CFM a en quelque sorte vécu en autarcie, et dans un isolement complet pendant la guerre, en exploitant la forêt comme n'importe lequel de ses concurrents. L'essentiel de ses ressources provenait de ventes d'okoumé (et d'ozigo) et de bois divers à l'exportation. A partir de cette date (1964), l'épuisement des ressources en okoumé et les investissements d'une deuxième scierie amenèrent à transférer l'activité essentiellement sur la vente de sciages, en particuliers de traverses et de longrines d'appareils. L'activité de menuiserie, à partir de 1965, permit d'absorber une partie non négligeable des frais généraux.

Quoi qu'il en soit, la période de 1964 à 1971 supporta une perte importante compensée par la trésorerie de la SNCF. La réalisation en fin de concession des actifs du CFM s'est déroulée dans d'excellentes conditions et a permis de rendre excédentaires les deux derniers exercices(1973 et 1974).

Au 31 décembre 1972, le total des pertes était de 877 millions de francs CFA compensé par un bénéfice pendant la période de liquidation de 273,6 millions de francs CFA.

Il en est donc résulté une perte globale de l'ordre de 600 millions de francs $\mathrm{CFA}$, soit 12 millions de francs français. Mais, pendant cette période, le CFM a versé à la SNCF 442 millions de francs CFA et a amorti dans ses comptes 1220 millions de francs CFA correspondant aux investissements.

On peut donc apprécier à 600 ou 158 millions de francs CFA la perte totale. Si l'on considère que pendant cette période le CFM a livré $146000 \mathrm{~m}^{3}$ de sciages à la SNCF, il en résulte un surcoût de 2 à 8 francs français par traverse sur un prix moyen de 40 francs ; encore ce chiffre ne tient-il pas compte des bois d'appareils dont le prix unitaire était nettement plus élevé. Le CFM a finalement permis un approvisionnement régulier et de qualité à des prix compétitifs.

8- Le « franc des colonies françaises d'Afrique » a été créé le 25 décembre 1945, avec une parité de 1,7 avec le franc français, de 2 en octobre 1948, de 0,02 en 1960 (nouveau franc) jusqu'à la dévaluation du 11 janvier 1994, la parité s'établissant alors à $0,01 \mathrm{FRF}$. Son cours par rapport à l'euro a été fixé en 1998 à 1 euro $=6555,957$ francs CFA (N.d.l.R.). 